\title{
The Gibbs phenomenon for Stromberg wavelets
}

\author{
By Vazgen Gagik MIKAYELYAN \\ Faculty of Mathematics and Mechanics, Yerevan State University, \\ 1 Alex Manoogian, Yerevan 0025, Republic of Armenia \\ (Communicated by Kenji Fukaya, M.J.A., Feb. 12, 2020)
}

\begin{abstract}
The Gibbs phenomenon for Stromberg wavelets is studied. It is proved that the Gibbs phenomenon for partial sums of Fourier-Stromberg series occurs for almost all points of $\mathbf{R}$.
\end{abstract}

Key words: Gibbs phenomenon; wavelets; Stromberg systems.

The Gibbs phenomenon, discovered by Henry Wilbraham in 1848 and rediscovered by Josiah Willard Gibbs in 1899, is the peculiar manner in which the Fourier series of some function behaves at a jump discontinuity. The $n$-th partial sum of the Fourier series has large oscillations near the jump, which might increase the maximum of the partial sum above that of the function itself. The overshoot does not die out as $n$ increases, but approaches a finite limit.

Modifying the Franklin system, Strömberg [11] obtained a system, which is an unconditional basis in $H^{p}(\mathbf{R})$, for $p>\frac{1}{2}$, where the space $H^{p}(R)$ is the boundary values on the real axis of the real parts of those analytic functions $F$ in the upper half-plane which satisfy

$$
\sup _{y>0} \int_{-\infty}^{\infty}|F(x+i y)|^{p} d x<\infty .
$$

Let $m \geq 0, A_{0}=\mathbf{N} \cup\{0\} \cup \frac{1}{2}(-\mathbf{N})$ and $A_{1}=$ $A_{0} \cup\left\{\frac{1}{2}\right\}$, where $\mathbf{N}$ is the set of natural numbers. The points of the set $A_{0}$ split $\mathbf{R}$ into intervals $\left\{I_{\sigma}\right\}_{\sigma \in A_{0}}$, where $\sigma$ is the left endpoint of the interval $I_{\sigma}$. Let $S_{0}^{m}$ be the subspace of functions $f \in L^{2}(\mathbf{R})$, such that $f \in L^{2}(\mathbf{R}) \cap C^{m}(\mathbf{R})$ and $f$ is a real polynomial of degree not greater than $m+1$ on each $I_{\sigma}, \sigma \in A_{0}$. Let $S_{1}^{m}$ be the corresponding subspace of $L^{2}(\mathbf{R})$ with the set $A_{0}$ replaced by $A_{1}$. It is clear that $S_{0}^{m} \subset S_{1}^{m}$ and if $f(x) \in S_{0}^{m}$, then $f(x-1) \in S_{1}^{m}$. In general, the functions of $S_{1}^{m}$ differ from the functions of $S_{0}^{m}$ only by the following: a function from $S_{0}^{m}$ is a polynomial on $[0,1]$, while a

2010 Mathematics Subject Classification. Primary 42C40; Secondary $42 \mathrm{C} 10$. function from $S_{1}^{m}$ can be polynomial only at each of the intervals $\left[0, \frac{1}{2}\right],\left[\frac{1}{2}, 1\right]$. It follows that $S_{0}^{m}$ had codimension 1 in $S_{1}^{m}$. Therefore there exists a function $f^{(m)} \in L^{2}(\mathbf{R})$, that is uniquely defined (up to the sign) by the following relations:

1. $f^{(m)} \in S_{1}^{m}$,

2. $f^{(m)} \perp S_{0}^{m}$, that is $\int_{\mathbf{R}} f^{(m)}(x) f(x) d x=0$ for all $f \in S_{0}^{m}$,

3. $\left\|f^{(m)}\right\|_{2}=1$.

For all pairs $(i, j) \in \mathbf{Z}^{2}$ denote

$$
f_{i, j}^{(m)}(x)=2^{\frac{i}{2}} f^{(m)}\left(2^{i} x-j\right) .
$$

The system $\left\{f_{i, j}^{(m)}(x)\right\}_{i, j \in \mathbf{Z}}$ was introduced by Strömberg in [11]. He also proved that $\left\{f_{i, j}^{(m)}(x)\right\}_{i, j \in \mathbf{Z}}$ is a complete orthonormal system in $L^{2}(\mathbf{R})$ and it is an unconditional basis in $H^{p}(\mathbf{R})$ for all $p>\frac{1}{m+2}$.

Let $t_{0}$ be a discontinuity point of the first kind of a function $q \in L(\mathbf{R})$, such that $\mid q\left(t_{0}+\right)-$ $q\left(t_{0}-\right) \mid=2 d>0$, and let $\left\{q_{i, j}(t)\right\}_{i, j \in \mathbf{Z}}$ be any sequence of functions converging to $q(t)$ at every point of some neighborhood of $t_{0}$, when $i, j \rightarrow+\infty$. Then the value of the Gibbs function for sequence $\left\{q_{i, j}(t)\right\}_{i, j \in \mathbf{Z}}$ at $t_{0}$ is defined by

$$
\begin{aligned}
& G\left(t_{0}, q,\left\{q_{i, j}\right\}_{i, j \in \mathbf{Z}}\right)=G\left(t_{0}\right) \\
& \quad=\varlimsup_{\substack{t \rightarrow t_{0} \\
i, j \rightarrow+\infty}} \frac{1}{d}\left|q_{i, j}(t)-\frac{q\left(t_{0}+\right)+q\left(t_{0}-\right)}{2}\right| .
\end{aligned}
$$

If $G\left(t_{0}\right)>1$, we say that the sequence $\left\{q_{i, j}(t)\right\}_{i, j \in \mathbf{Z}}$ exhibits the Gibbs phenomenon at $t_{0}$.

The Gibbs phenomenon has been studied for Fourier series with respect to the trigonometric system (see [2], pp. 123-126). In this case the value $G\left(t_{0}\right)$ is independent of $t_{0}$ and it is equal to the 
Gibbs constant:

$$
G\left(t_{0}\right)=\frac{2}{\pi} \int_{0}^{\pi} \frac{\sin t}{t} d t \approx 1.17 .
$$

The Gibbs phenomenon for Fourier series with respect to the classical Franklin system has been studied in [10]. It is proved that the Gibbs phenomenon occurs everywhere and the function $G$ is a constant almost everywhere. The case of the general Franklin system has been studied in [8]. It has been proved that the Gibbs phenomenon occurs almost everywhere.

The Gibbs phenomenon for Fourier series with respect to the Walsh system has been studied in the papers [12] and [1]. The existence of the Gibbs phenomenon for Fourier series with respect to Walsh system was established in [12]. In this case $G$ is not a constant. In [1] exact upper and lower bounds for this function are found.

Such problems are also studied in $[4,6]$.

Let

$$
\begin{gathered}
S_{i_{0}, j_{0}}^{(m)}(f, x)=\sum_{i=-\infty}^{i_{0}-1} \sum_{j=-\infty}^{+\infty} a_{i, j} f_{i, j}^{(m)}(x) \\
+\sum_{j=-\infty}^{j_{0}} a_{i_{0}, j} f_{i_{0}, j}^{(m)}(x), i_{0}, j_{0} \in \mathbf{Z}
\end{gathered}
$$

be the partial sum of Fourier-Stromberg series and let

$$
G\left(t_{0}, m\right)=G\left(t_{0}, f,\left\{S_{i_{0}, j_{0}}^{(m)}(f, \cdot)\right\}_{i_{0}, j_{0}=-\infty}^{+\infty}\right)
$$

be the Gibbs function. Also denote

$$
\begin{gathered}
K_{i_{0}, j_{0}}^{(m)}(x, t)=\sum_{i=-\infty}^{i_{0}-1} \sum_{j=-\infty}^{+\infty} f_{i, j}^{(m)}(x) f_{i, j}^{(m)}(t) \\
+\sum_{j=-\infty}^{j_{0}} f_{i_{0}, j}^{(m)}(x) f_{i_{0}, j}^{(m)}(t), i_{0}, j_{0} \in \mathbf{Z} .
\end{gathered}
$$

It is easy to see that for all bounded functions $f$ : $\mathbf{R} \rightarrow \mathbf{R}$

$$
S_{i_{0}, j_{0}}^{(m)}(f, x)=\int_{-\infty}^{+\infty} K_{i_{0}, j_{0}}^{(m)}(x, t) f(t) d t .
$$

In the case $m=0$ the Gibbs phenomenon has been studied in [9], where the following theorem was proved:

Theorem 1. Let $m=0$. If $t_{0}$ is a discontinuity point of the first kind of a function $f \in L^{2}(\mathbf{R})$, then the Gibbs phenomenon occurs everywhere in $\mathbf{R}$, and for all $t_{0} \in \mathbf{R}$

$$
\begin{gathered}
1+\frac{48-28 \sqrt{3}+8 \sqrt{2}(2-\sqrt{3})}{27} \\
\leq G\left(t_{0}, 0\right) \leq \frac{1+2 \sqrt{3}}{3},
\end{gathered}
$$

with $G\left(t_{0}, 0\right)=\frac{1+2 \sqrt{3}}{3}$ almost everywhere.

The main result of the present paper is the following theorem:

Theorem 2. Let $m \geq 0$. If $t_{0}$ is a discontinuity point of the first kind of a function $f \in L^{2}(\mathbf{R})$, then the Gibbs phenomenon occurs almost everywhere in $\mathbf{R}$, i.e. $G\left(t_{0}, m\right)>1$ for almost all $t_{0} \in \mathbf{R}$.

Let's prove the following lemma:

Lemma 1. There exists a natural number $k$, such that

$$
\int_{-\infty}^{k} K_{0,0}^{(m)}(k, t) d t>1 .
$$

Proof. Since from (2) we have

$$
\int_{-\infty}^{+\infty} K_{0,0}^{(m)}(k, t) d t=1,
$$

it suffices to prove that there exists a natural number $k$, such that

$$
\int_{k}^{+\infty} K_{0,0}^{(m)}(k, t) d t<0 .
$$

Recall, that B-spline with real knots $x_{0} \leq x_{1} \leq$ $\ldots \leq x_{r}$ is the following function

$$
M(x)=r\left[x_{0}, x_{1}, \ldots, x_{r}\right](\cdot-x)_{+}^{r-1}, x \in \mathbf{R},
$$

where square brackets mean divided differences (see [3], ch. 5, p. 2). For every $n \in A_{1}$ denote by $N_{n}(t)$ B-spline with $m+3$ consecutive knots from $A_{1}$, with starting knot $n$.

We have $K_{0,0}^{(m)}(1, t)=\sum_{n \in A_{1}} c_{n} N_{n}(t)$ and from (2) follows that

$$
\int_{-\infty}^{+\infty} K_{0,0}^{(m)}(1, t) N_{i}(t) d t=N_{i}(1)=0,
$$

for all $i \in \mathbf{N}$. Therefore 


$$
\sum_{n \in A_{1}} c_{n} \int_{-\infty}^{+\infty} N_{n}(t) N_{i}(t) d t=0
$$

for all $i \in \mathbf{N}$. Since $\int_{-\infty}^{+\infty} N_{n}(t) N_{i}(t) d t=0$, for $n+$ $m+2 \leq i$ and for $n \geq i+m+2$, we have

$$
\sum_{n=i-m-1}^{i+m+1} c_{n} \int_{-\infty}^{+\infty} N_{n}(t) N_{i}(t) d t=0
$$

for all $i \in \mathbf{N}$. We regard (3) as a recurrence relation with respect to $c_{i}$. The characteristic polynomial of (3) has order $2 m+2$. It is clear that 0 is not a root of that polynomial and if $x_{0}$ is its root, then $\frac{1}{x_{0}}$ is also its root. Since $\lim _{i \rightarrow+\infty} c_{i}=0$ (see [11]), for all $i \in$ $\mathbf{N}$

$$
c_{i}=\sum_{j=1}^{t} \alpha_{j}^{i}\left(a_{j, 1}+a_{j, 2} i+\ldots+a_{j, n_{j}-1} i^{n_{j}-1}\right),
$$

where $\alpha_{1}, \ldots, \alpha_{t}$ are those roots of the characteristic polynomial which by modulo are less than one and $n_{j}$ is the multiplicity of the root $\alpha_{j}$ and the coefficients $a_{j, h}$ are real (see [5], Section 3.3). Denote

$$
d_{j}=\int_{1}^{j+2} N_{1}(t) d t
$$

for all $j \in\{0,1, \ldots, m\}$.

Let $\left|\alpha_{1}\right|=\max \left\{\left|\alpha_{1}\right|,\left|\alpha_{2}\right|, \ldots,\left|\alpha_{t}\right|\right\}$. It is clear that if $\alpha_{1} \in \mathbf{R}$, then

$$
c_{i}=a_{1, n_{1}-1} i^{n_{1}-1} \alpha_{1}^{i}+o\left(i^{n_{1}-1}\left|\alpha_{1}\right|^{i}\right),
$$

and if $\alpha_{1} \in \mathbf{C} \backslash \mathbf{R}$, then

$$
c_{i}=2 \operatorname{Re}\left(a_{1, n_{1}-1} i^{n_{1}-1} \alpha_{1}^{i}\right)+o\left(i^{n_{1}-1}\left|\alpha_{1}\right|^{i}\right) .
$$

If $k>m+3$ and $k \in \mathbf{N}$, then

$$
\begin{aligned}
\int_{k}^{+\infty} K_{0,0}^{(m)}(1, t) d t=\sum_{n \in A_{1}} c_{n} \int_{k}^{+\infty} N_{n}(t) d t \\
=\sum_{n=k-m-1}^{+\infty} c_{n} \int_{k}^{+\infty} N_{n}(t) d t \\
=\sum_{n=k-m-1}^{k-1} c_{n} \int_{k}^{+\infty} N_{n}(t) d t+\sum_{n=k}^{+\infty} c_{n}
\end{aligned}
$$

$$
=\sum_{l=0}^{m} c_{l+k-m-1} d_{l}+\sum_{n=k}^{+\infty} c_{n} .
$$

Hence the sign of the number $\int_{k}^{+\infty} K_{0,0}^{(m)}(1, t) d t$ coincides with the sign of the following number

$$
\begin{aligned}
\operatorname{Re}( & \sum_{l=0}^{m} a_{1, n_{1}-1} \alpha_{1}^{l+k-m-1}(l+k-m-1)^{n_{1}-1} d_{l} \\
& \left.+\sum_{n=k}^{+\infty} a_{1, n_{1}-1} \alpha_{1}^{n} n^{n_{1}-1}\right)+o\left(k^{n_{1}-1}\left|\alpha_{1}\right|^{k}\right) \\
= & \operatorname{Re}\left(\alpha_{1}^{k-m-1}(k-m-1)^{n_{1}-1} a_{1, n_{1}-1} x_{k}\right. \\
& \left.+a_{1, n_{1}-1} \alpha_{1}^{k} k^{n_{1}-1} z_{k}\right)+o\left(k^{n_{1}-1}\left|\alpha_{1}\right|^{k}\right) \\
= & \operatorname{Re}\left(\alpha_{1}^{k-m-1}(k-m-1)^{n_{1}-1} a_{1, n_{1}-1} x_{k}\right) \\
& +o\left(k^{n_{1}-1}\left|\alpha_{1}\right|^{k}\right),
\end{aligned}
$$

where

$$
\begin{aligned}
x_{k}= & \sum_{l=0}^{m} \alpha_{1}^{l}\left(1+\frac{l}{k-m-1}\right)^{n_{1}-1} d_{l} \\
& +\frac{\alpha_{1}^{m+1}}{1-\alpha_{1}}\left(1+\frac{m+1}{k-m-1}\right)^{n_{1}-1}
\end{aligned}
$$

and

$$
z_{k}=\sum_{n=0}^{+\infty} \alpha_{1}^{n}\left(1+\frac{n}{k}\right)^{n_{1}-1}-\frac{1}{1-\alpha_{1}} .
$$

For obtaining the formula (4) we used that $\lim _{k \rightarrow \infty} z_{k}=$ 0 . Suppose there exists $\beta \in\left\{0,1, \ldots, n_{1}-1\right\}$, for which there exists a finite nonzero limit $\lim _{k \rightarrow+\infty}(k-m-1)^{\beta} x_{k}$. Denote $y_{k}=(k-m-1)^{\beta} x_{k}$, $y=\lim _{k \rightarrow+\infty} y_{k}, \quad \psi_{k}=\arg y_{k}, \quad \psi_{0}=\arg y, \quad \varphi=\arg \alpha_{1}$, $\varphi_{0}=\arg a_{1, n_{1}-1}$. We can assume that $\varphi \in(0, \pi]$. If $\varphi=\pi$, then $\alpha_{1}<0$; therefore

$$
\begin{aligned}
& \operatorname{Re}\left(\alpha_{1}^{k-m-1}(k-m-1)^{n_{1}-1} a_{1, n_{1}-1} x_{k}\right) \\
& \quad=\alpha_{1}^{k-m-1}(k-m-1)^{n_{1}-1-\beta} a_{1, n_{1}-1} y_{k} .
\end{aligned}
$$

Since $y \neq 0$, the expression above has different signs for $k$ and $k+1$. Therefore $\int_{k}^{+\infty} K_{0,0}^{(m)}(1, t) d t<0$ or $\int_{k+1}^{+\infty} K_{0,0}^{(m)}(1, t) d t<0$

Now let $\varphi \in(0, \pi)$. Note that 


$$
\begin{aligned}
\operatorname{Re}\left(\alpha_{1}^{k-m-1}(k-m-1)^{n_{1}-1-\beta} a_{1, n_{1}-1} y_{k}\right) \\
=\left|\alpha_{1}\right|^{k-m-1}(k-m-1)^{n_{1}-1-\beta}\left|a_{1, n_{1}-1}\right|\left|y_{k}\right| \\
\quad \cdot \cos \left((k-m-1) \varphi+\varphi_{0}+\psi_{k}\right) .
\end{aligned}
$$

We choose $\varepsilon>0$ such that $\frac{\pi-2 \varepsilon}{\varphi}>1$. Then for all $n \in \mathbf{N}$ the length of the segment

$$
\left[\frac{\frac{\pi}{2}+2 \pi n-\varphi_{0}-\psi_{0}+\varepsilon}{\varphi}, \frac{\frac{3 \pi}{2}+2 \pi n-\varphi_{0}-\psi_{0}-\varepsilon}{\varphi}\right]
$$

will be greater than one. Since $\lim _{k \rightarrow+\infty} \psi_{k}=\psi_{0}$, there exists $k_{0} \in \mathbf{N}$, such that $\psi_{k} \in\left(\psi_{0}-\varepsilon, \psi_{0}+\varepsilon\right)$ for all $k \geq k_{0}$. It follows that there are infinitely many $k$, such that

$$
\cos \left((k-m-1) \varphi+\varphi_{0}+\psi_{k}\right)<0 .
$$

Now suppose that for no $\beta \in\{0,1, \ldots, n-1\}$, there exists a finite nonzero $\operatorname{limit} \lim _{k \rightarrow+\infty}(k-m-$ $1)^{\beta} x_{k}$. It is clear that in this case $x_{k}=0$, for $k>m+3$. Hence taking into account (4) it suffices to prove that the number

$$
\operatorname{Re}\left(a_{1, n_{1}-1} \alpha_{1}^{k} k^{n_{1}-1} z_{k}\right)+o\left(k^{n_{1}-1}\left|\alpha_{1}\right|^{k}\right),
$$

has a negative sign. Suppose $n_{1} \neq 1$. It is clear that in this case there exists a finite nonzero limit $\lim _{k \rightarrow+\infty} k z_{k}$. Hence, in the same way, the desired result can be obtained.

Now suppose $n_{1}=1$. Then we will change the form $c_{i}$ in the following way

$$
c_{i}=a_{1,0} \alpha_{1}^{i}+a_{1, n_{2}-1} i^{n_{2}-1} \alpha_{2}^{i}+o\left(i^{n_{2}-1}\left|\alpha_{2}\right|^{i}\right),
$$

where $\left|\alpha_{2}\right|=\max \left\{\left|\alpha_{2}\right|,\left|\alpha_{3}\right|, \ldots,\left|\alpha_{t}\right|\right\}$. Similarly, we find that it suffices to investigate the case $n_{2}=1$. Doing the same steps, we get that we can assume $n_{1}=n_{2}=\ldots=n_{t}=1$. We got that $x_{k}=0$, if $k>$ $m+3$, for $\alpha_{1}$; in particular $\lim _{k \rightarrow \infty} x_{k}=0$. Consequently, the following identity holds

$$
\sum_{l=0}^{m} \alpha_{1}^{l} d_{l}+\frac{\alpha_{1}^{m+1}}{1-\alpha_{1}}=0 .
$$

This is equivalent to that $\alpha_{1}$ is a root of the polynomial

$$
\begin{aligned}
& P(x)=d_{0}+\left(d_{1}-d_{0}\right) x+\left(d_{2}-d_{1}\right) x^{2}+\ldots \\
& \quad+\left(d_{m}-d_{m-1}\right) x^{m}+\left(1-d_{m}\right) x^{m+1} .
\end{aligned}
$$

In the same way we get that $P\left(\alpha_{i}\right)=0$, for all $i \in$
$\{1,2, \ldots, m+1\}$. From definitions of $d_{0}, d_{1}, \ldots, d_{m}$ it follows that $P\left(\frac{1}{\alpha_{i}}\right)=0$, for all $i \in 1,2, \ldots$, $m+1$. Since $\left|\alpha_{i}\right|<1$, for $i \in 1,2, \ldots, m+1$, the polynomial $P$ will have $2 m+2$ different zeros, which contradicts the fact that $P$ has order $m+1$.

Lemma 2. If $\alpha, \beta(\alpha<\beta)$ are real numbers and if $A_{i, j}=\left(\frac{\alpha+j}{2^{i}}, \frac{\beta+j}{2^{i}}\right), i, j \in \mathbf{Z}$, then

$$
\mu\left(\mathbf{R} \backslash \bigcap_{l=1}^{+\infty} \bigcup_{i=l}^{+\infty} \bigcup_{j \in \mathbf{Z}} A_{i, j}\right)=0,
$$

where $\mu$ is a Lebesgue measure.

Proof. Let $l \in \mathbf{N}$. Since the sequence $\left\{2^{n} x\right\}$, $n \in \mathbf{N}, n \geq l$ is everywhere dense in $[0,1]$ for almost all $x \in[0,1]$ (see [7], problems 4.3, 1.6), for almost all $x \in \mathbf{R}$ there exists $i \geq l$ such that $x \in A_{i,\left[{ }^{2} i x\right]}$. It follows that

$$
\mu\left(\mathbf{R} \backslash \bigcup_{i=l}^{+\infty} \bigcup_{j \in \mathbf{Z}} A_{i, j}\right)=0,
$$

for all $l \in \mathbf{N}$, which ends the proof.

Proof of theorem 2. From (2) and lemma 1 follows that there exist constants $\varepsilon, \delta>0$ and $k_{0} \in$ $\mathbf{N}$ such that $S_{0,0}^{(m)}\left(\varphi_{x}, k_{0}\right)>1+\delta$ for all $x \in\left(k_{0}-\varepsilon\right.$, $\left.k_{0}+\varepsilon\right)$, where

$$
\varphi_{x}(t)=\left\{\begin{array}{l}
1, t \leq x \\
0, t>x
\end{array}\right.
$$

Denote $I_{i, j}=\left(\frac{k_{0}-\varepsilon+j}{2^{i}}, \frac{k_{0}+\varepsilon+j}{2^{i}}\right), i, j \in \mathbf{Z}$. From (1) we obtain

$$
K_{i, j}\left(k_{0}, t\right)=2^{i} K_{0,0}\left(2^{i} k_{0}-j, 2^{i} t-j\right) ;
$$

thus

$$
S_{i, j}^{(m)}\left(\varphi_{x}, \frac{k_{0}+j}{2^{i}}\right)>1+\delta, x \in I_{i, j} .
$$

Therefore from lemma 2 follows that for almost all $x \in \mathbf{R}$ there exist sequences $i_{n}, j_{n}$ such that $\lim _{n \rightarrow \infty} \frac{k_{0}+j_{n}}{2^{i_{n}}}=x$ and

$$
S_{i_{n}, j_{n}}^{(m)}\left(\varphi_{x}, \frac{k_{0}+j_{n}}{2^{i_{n}}}\right)>1+\delta, x \in I_{i, j} .
$$

It follows that

$$
\varlimsup_{\substack{t \rightarrow x \\ i, j \rightarrow+\infty}} S_{i, j}^{(m)}\left(\varphi_{x}, t\right)>1+\delta .
$$

To complete the proof it remains to do the following steps as in [9]: 
1) Prove that if $g$ is an integrable function on $\mathbf{R}$ which is continuous at $x$, then

$$
\lim _{\substack{t \rightarrow x \\ i, j \rightarrow+\infty}} S_{i, j}^{(m)}(g, t)=g(x) .
$$

The main part of proof of this point, is that for all $x \in \mathbf{R}$ and $\delta>0$

$$
\lim _{\substack{t \rightarrow x \\ i, j \rightarrow+\infty}} \int_{|s-x| \geq \delta}\left|K_{i, j}^{(m)}(t, s)\right| d s=0 .
$$

2) Prove that $G(t, m)>1+\delta$ for almost all $t \in \mathbf{R}$. Proof of this fact for characteristic functions follows from (5).

Acknowledgement. I would like to thank my supervisor Prof. G. G. Gevorkyan for his assistance to this research and valuable comments.

\section{References}

[ 1 ] L. A. Balashov and V. A. Skvortsov, Gibbs constants for partial sums of Fourier-Walsh series and their $(C, 1)$-means, Trudy Mat. Inst. Steklov. 164 (1983), 37-48.

[ 2 ] N. K. Bari, Trigonometric series, Gosudarstv. Izdat. Fiz.-Mat. Lit., Moscow, 1961. (in Russian).

[ 3 ] R. A. DeVore and G. G. Lorentz, Constructive approximation, Grundlehren der Mathematischen Wissenschaften, 303, Springer-Verlag, Berlin, 1993.

[ 4 ] C. Karanikas, Gibbs phenomenon in wavelet analysis, Results Math. 34 (1998), no. 3-4, 330-341.

[ 5 ] W. G. Kelley and A. C. Peterson, Difference Equations: An Introduction with Applications, 2nd ed., Academic Press, New York, 2001.

[ 6 ] S. E. Kelly, Gibbs phenomenon for wavelets, Appl. Comput. Harmon. Anal. 3 (1996), no. 1, $72-81$.

[ 7 ] L. Kuipers and H. Niederreiter, Uniform distribution of sequences, Wiley-Interscience, New York, 1974.

[ 8 ] V. G. Mikaelyan, The Gibbs phenomenon for general Franklin systems, J. Contemp. Math. Anal. 52 (2017), no. 4, 198-210; translated from Izv. Nats. Akad. Nauk Armenii Mat. 52 (2017), no. $4,51-71$.

[ 9 ] V. G. Mikaelyan, The Gibbs phenomenon for Stromberg's piecewise linear wavelet, J. Contemp. Math. Anal. 54 (2019), no. 2, 112-123; translated from Izv. Nats. Akad. Nauk Armenii Mat. 54 (2019), no. 2, 65-81.

[ 10 ] O. G. Sargsyan, On the convergence and Gibbs phenomenon of Franklin series, J. Contemp. Math. Anal. 31 (1996), no. 1, 51-72; translated from Izv. Nats. Akad. Nauk Armenii Mat. 31 (1996), no. 1, 61-84.

[11 J.-O. Strömberg, A modified Franklin system and higher-order spline systems on $\mathbf{R}^{n}$ as unconditional bases for Hardy spaces, in Conference on harmonic analysis in honor of Antoni Zygmund, (Chicago, 1981), 475-494, Wadsworth Math. Ser., Wadsworth, Belmont, CA, 1983.

[ 12 ] A. M. Zubakin, The Gibbs phenomenon for multiplicative systems of Walsh or VilenkinDzhofarli type, Siberian Math. J., 12 (1971), no. 1, 147-157. 